
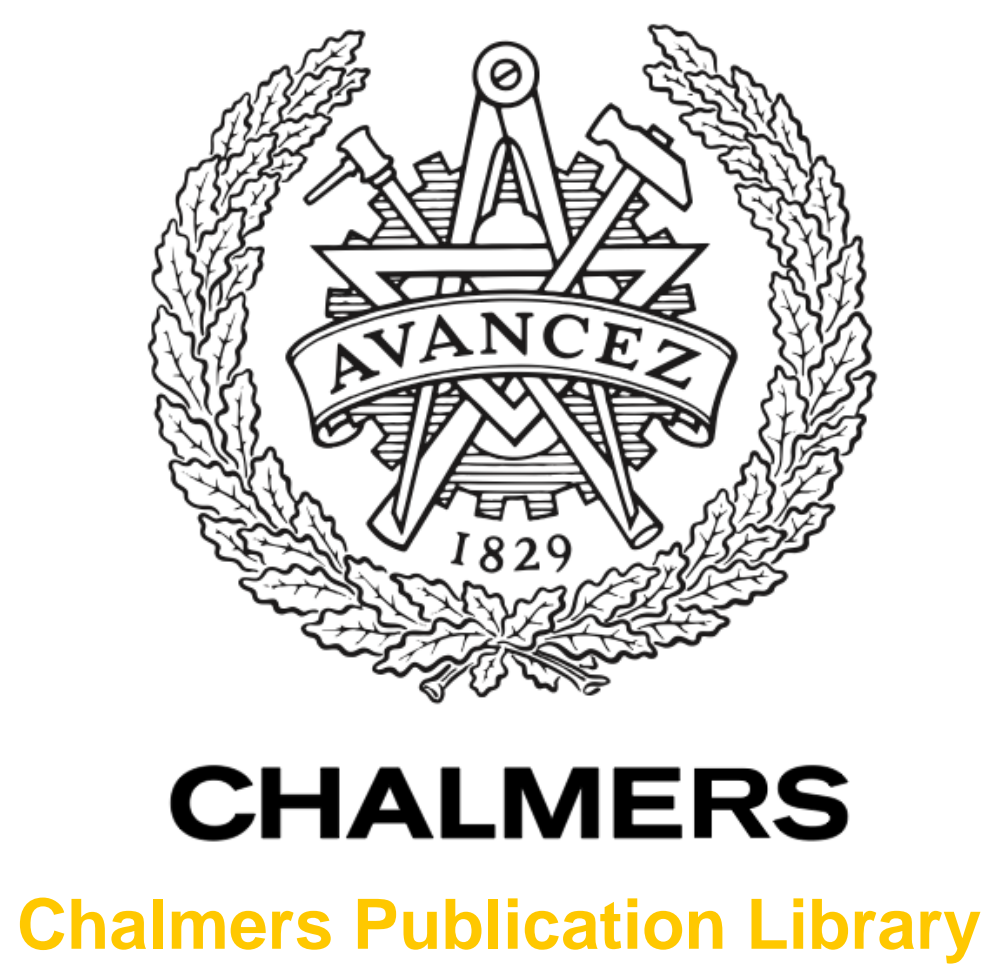

Idler chirp optimization in a pulse-pumped parametric amplifier

This document has been downloaded from Chalmers Publication Library (CPL). It is the author's version of a work that was accepted for publication in:

2013 IEEE Photonics Society Summer Topical Meeting Series, PSSTMS 2013

Citation for the published paper:

Wiberg, A. ; Tong, Z. ; Myslivets, E. (2013) "Idler chirp optimization in a pulse-pumped parametric amplifier". 2013 IEEE Photonics Society Summer Topical Meeting Series, PSSTMS 2013 pp. 159-160.

http://dx.doi.org/10.1109/PHOSST.2013.6614518

Downloaded from: http://publications.lib.chalmers.se/publication/187556

Notice: Changes introduced as a result of publishing processes such as copy-editing and formatting may not be reflected in this document. For a definitive version of this work, please refer to the published source. Please note that access to the published version might require a subscription.

Chalmers Publication Library (CPL) offers the possibility of retrieving research publications produced at Chalmers University of Technology. It covers all types of publications: articles, dissertations, licentiate theses, masters theses, conference papers, reports etc. Since 2006 it is the official tool for Chalmers official publication statistics. To ensure that Chalmers research results are disseminated as widely as possible, an Open Access Policy has been adopted.

The CPL service is administrated and maintained by Chalmers Library. 


\title{
Idler Chirp Optimization in a Pulse-Pumped Parametric Amplifier
}

\author{
A.O.J. Wiberg ${ }^{1}$, Z. Tong, E. Myslivets, N. Alic and \\ S. Radic \\ Department of Electrical and Computer Engineering, \\ University of California San Diego, \\ 9500 Gilman Drive, La Jolla, CA 92093-0407 \\ 1e-mail: awiberg@ucsd.edu
}

\begin{abstract}
A simple engineering rule for idler characteristic optimization in pulsed fiber optic parametric amplifiers operated in transparency is derived. The theoretical results are validated in both simulations and experiments.
\end{abstract}

Keywords - Parametric processing, Nonlinear optics, fourwave mixing, parametric sampling, chirp

\section{INTRODUCTION}

Fiber optical parametric amplifiers (FOPAs) are a proven, mature platform in all-optical signal processing that has been demonstrated as highly effective in a wide range of applications, spanning from continuous-wave (CW) pumped high-gain amplification, to wavelength conversion, as well as processing of both analog and digital signals [1]. Furthermore, FOPAs have been successfully used for short pulse generation taking advantage of their exponential transfer characteristic in a high gain regime [2]. In many applications, such as high-speed signal processing, optical sampling and pulse generation, a pulsed pump is used to convert a temporal fraction of the signal, to a new wavelength, i.e. the idler. The generated idler is proportional to the signal shape - gated with the FOPA conversion characteristics. Flat and broadband response together with spectral-shape control of the generated idler is of

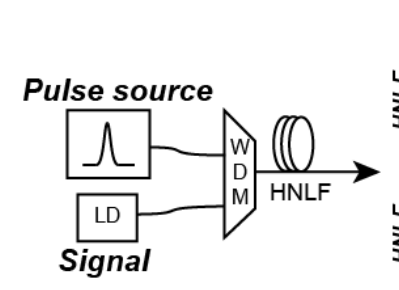

(a)
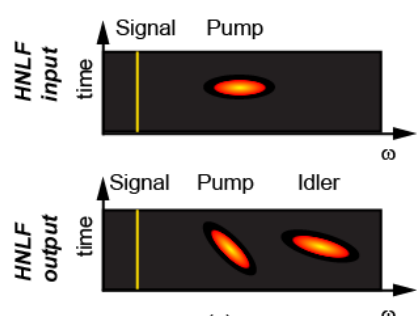

(c)

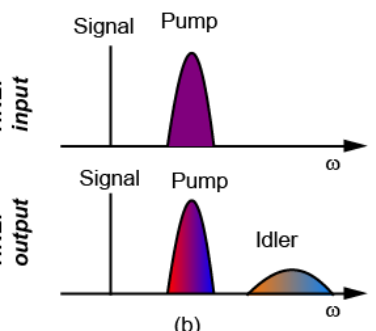

(b)
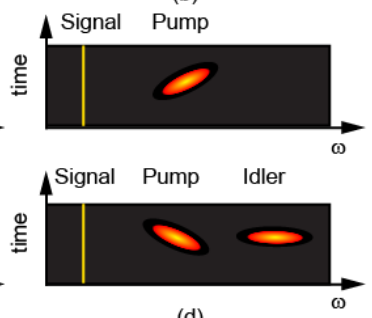

(d)

Fig. 1. (a) Overview of a FOPA, (b) spectra at the in and output of the FOPA, time and frequency illustration of in- and output of the FOPA with (c) initial chirp-free pump and (d) initial negative chirped pump.

\author{
M. Karlsson \\ Department of Microtechnology and Nanoscience \\ Chalmers University of Technology \\ Gothenburg, Sweden
}

primary interest in sampling and high fidelity wavelength tunable pulse generation $[3,4]$. However, in this mode of operation the FOPA gain characteristic varies significantly with wavelength, which can limit the wavelength tuning range of the pulse-source. Moreover, FOPA operation in a high gain regime also introduces a significant pump noise transfer in addition to the ever present amplified quantum noise [5].

In this contribution we investigate properties of a pulsepumped FOPA, and in particular the chirp characteristics of the pump and idler. Specifically, the main focus of the investigation is on a FOPA operating in, or near transparency (i.e. a unity conversion efficiency), where low noise and broadband spectral control are possible. In a polychromatic sampling operation [4], where a single pulsed pump is simultaneously sampling several signal copies at different wavelengths, optimization of the spectral width decreases the spectral overlap of the sampled copies and, thus, improves the performance of the sampling gate. Minimizing the spectral width, in turn, is equivalent to attaining a chirp-free pulse. Consequently our investigation is aimed at finding the optimal initial pump condition leading to a chirp-free idler generation, when the FOPA is operated at transparency.

\section{CHIRP IN PARAMETRIC AMPLIFIER}

A pulse-pumped FOPA with a CW input signal is depicted in Fig 1. (a) and (b). It is well known that the high power pump pulse will induce a self-inflicted chirp via self-phase modulation (SPM) as it propagates through an optical fiber [6]. The output chirp of the pump $\left(C_{\mathrm{P}, \text { out }}\right)$ is dependent on the input chirp $\left(C_{\mathrm{P}, \mathrm{in}}\right)$, that is captured by the following relationship:

$$
C_{\mathrm{P}, \text { out }}=2 \gamma P_{\mathrm{p}} L+C_{\mathrm{P}, \text { in }}
$$

where $\gamma$ is the nonlinear coefficient of the fiber, $P_{\mathrm{p}}$ is the pump peak power and $L$ is the length of the fiber and the dimensionless chirp factor, $C$, of a pulse is defined by

$$
C=-\frac{d^{2} \phi}{d t^{2}} T_{0}^{2} .
$$

A Gaussian pulse shape of width $\mathrm{T}_{0}$ and phase, $\phi$, as defined in e.g. $[2,6]$ is assumed throughout. In a high gain regime, the idler chirp is also dependent on frequency offset from the pump [2]. Here, we particularly consider the pump center wavelength as being positioned exactly at the zero dispersion wavelength (ZDW) in order to achieve an ultra-broadband 
operation of the sampling gate. Furthermore, the pulsed FOPA is to be operated in transparency (i.e. $\gamma P_{\mathrm{p}} L=1$ ) to minimize the pump noise transfer in the idler generation [1]. The generated idler pulse transform limited characteristic (i.e. chirp-free) is considered beneficial in a FOPA used for pulse generation (or optical sampling), since its spectral width is simultaneously minimized. In correspondence to the transform limited input pump acquiring chirp in a FOPA the idler is generally characterized by a finite amount of chirp which depends on the pump power and the input chirp. Generated idler chirp is illustrated in the spectrogram in Fig.1(c). As implied by the interactions above, the pump-dependent idler chirp can be controlled by pump pre-chirping. In consequence, under proper conditions, a chirp-free idler can be created at the FOPA output, as depicted in Fig.1(d). As stemming from the theory in [2], an analytical solution of the idler chirp can be derived by accounting for an initial pump chirp $C_{\mathrm{P} \text {,in }}$. The expression is greatly simplified in the special case when the pump wavelength is equal to the zero dispersion wavelength:

$$
C_{\text {idler }}=\gamma P_{\mathrm{p}} L+C_{\mathrm{P}, \text { in }} .
$$

Operating in the transparency region (i.e. when $\gamma P_{\mathrm{p}} L=1$ ), one can easily find that the idler chirp can be suppressed altogether for an initial pump chirp of -1. This straightforward prediction was further investigated by simulating a FOPA using a rigorous non-linear Schrödinger solver. In the simulation, a $10 \mathrm{~m}$ long HNLF was used, characterized with a ZDW of $1561 \mathrm{~nm}$, a dispersion slope of $0.02 \mathrm{ps} / \mathrm{nm}^{2} \mathrm{~km}$, and a nonlinear coefficient of $14 \mathrm{~W}^{-1} \mathrm{~km}^{-1}$. Pump peak power and pump initial chirp were used as parameters in the simulation, as defined above. The pump consisted of Gaussian pulses with varying chirp, a pulsewidth of 3.5 ps with a repetition rate of 2 $\mathrm{GHz}$ with a $\mathrm{CW}$ signal. The simulation results are presented in Fig.2, showing the optical spectra at the output of the fiber for a pump peak power of $7 \mathrm{~W}$ (giving a unity gain for the parameters above) and pump chirp factors $C_{\mathrm{P} \text {,in }}$ of $-1,0$, and 1 , respectively. The idler was compressed to $2.5 \mathrm{ps}$ in all cases, since the FOPA was operated in the quadratic gain regime [1]. More importantly, as can be clearly seen in Fig. 2, different initial pump chirp conditions result in different respective output pump and idler spectral widths. The last two observation (i.e. the time and frequency domains' signal shapes), clearly imply a change in the output pulse chirp. To further demonstrate the chirp variation, the corresponding chirp values of the idler and pump pulses are presented in contour plots in Fig.2(c) and 2(d) using the initial pump chirp factor $C_{\mathrm{P}, \text { in }}$ and pump peak power as parameters. The observed agreement with the analytical results (1) and (3) is excellent. A chirp-free region can be clearly identified in Fig.2(c). The contour plots clearly show that at transparency, a 7-W pump peak power should be used together with a pump chirp factor $C_{\mathrm{P} \text {,in }}$ of -1 , to achieve a chirp-free idler, as predicted by Eq. (1).

The finding was further confirmed experimentally, with a cavity-less pulse source [4] used as pump pulse generator. The pulse source was designed so as to generate chirped pulses that are subsequently compressed. In the experiment, a programmable dispersive element was used for temporal compression (and, thus, the generated pulses' chirp) control. Two sets of pulses having similar temporal pulse widths, but
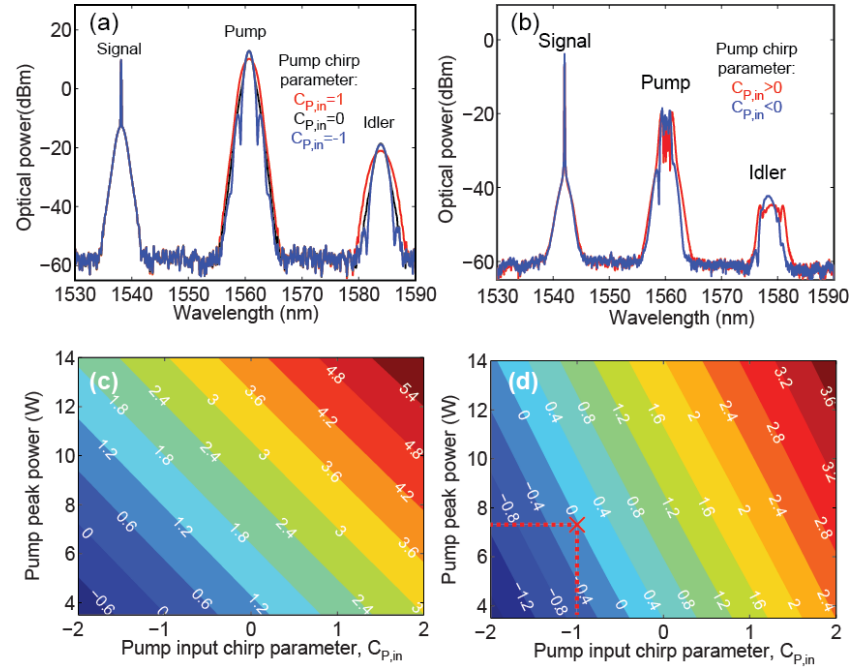

Fig. 2. Fig. 2 Spectral response of pulsed FOPA with different initial pump chirp (a) simulated and (b) experimental results. Contour plots of out put chirp of (c) pump and (d) idler as function of initial pump chirp and power.

opposite chirp signs were generated. The generated pulses were used as the pump in a FOPA, with HNLF parameters matching those in the simulation. The captured output spectra are shown in Fig.2(b), with spectral shapes after the HNLF changing according to the theoretical predictions, with a compressed idler spectrum achieved for a negative unity initial pump chirp.

\section{CONCLUSIONS}

We have demonstrated an idler spectral width optimization technique in FOPAs operated at transparency stemming from a fundamental four-wave mixing interaction. An engineering rule was derived from a simplified theory, dictating that pump pulses with a specific negative unity chirp lead to an optimal output condition. Moreover, the analytical predictions were fully validated both by simulations and experiments. The derived engineering rule bears significant implications on optical sampling in general, and particularly on polychromatic sampling, as well as, chirp-free pulse generation.

\section{ACKNOWLEDGMENT}

This material is based in part on research sponsored by Office of Naval Research (ONR). The authors acknowledge Sumitomo Electric Industries for providing the HNLF.

\section{REFERENCES}

[1] J. Hansryd et.al., IEEE Journal of Selected Topics in Quantum Electronics, vol.8, no.3, pp.506-520 (2002).

[2] T. Torounidis, et.al, Journal of Lightwave Technology, vol.23, no.12, pp. 4067- 4073 (2005).

[3] M. Westlund, et.al, J. Lightwave Technol. vol.23, 2012-2022 (2005).

[4] A. O. J. Wiberg et.al, Optical Fiber Communication Conference, paper OW3C.2, (2012)

[5] P. Kylemark, et.al, Lightwave Technology, Journal of, vol.22, no.2, pp. 409- 416 (2004).

[6] G.P. Agrawal, "Nonlinear Fiber Optics", fourth edition, Academic press, Burlington, MA, USA (2007). 